\title{
Research Article \\ Field and Temperature Gradients from Short Conductors in a Dissipative Medium
}

\author{
Quirino Balzano, Kenneth R. Foster, and Asher R. Sheppard
}

Received 31 March 2007; Accepted 28 October 2007

Recommended by Charles Bunting

\begin{abstract}
This paper considers the specific absorption rate (SAR) in tissue of radiofrequency (RF) energy and temperature increases produced by RF currents on short conductors $(0.03-0.1 \lambda)$. We consider a cylindrical model in which a center-feeds, insulated antenna is embedded in tissue. We introduce a new method for the analytic evaluation of the fields in the cylindrical phantom taking advantage of the axial symmetry of the antenna and the tissue. Results of the analytical model are compared to results of numerical (finite difference time domain) simulations; in addition, the thermal response of the exposed material is calculated by finite element solution of the heat conduction equation. For model antennas of 1 to $3 \mathrm{~cm}$ total length with a feedpoint current of $10 \mathrm{~mA}$ RMS at $900 \mathrm{MHz}$, the maximum SAR (in tissue next to the antenna) is less than $\sim 2.5 \mathrm{~W} / \mathrm{kg}$. SAR decays rapidly with radial distance from the antenna $\left(\sim r^{-4}\right.$ for the $1 \mathrm{~cm}$ antenna) and creates a steady-state temperature rise less than $0.05 \mathrm{~K}$ at the location of $\mathrm{SAR}_{\max }$. Heat conduction causes the temperature to decline steeply with radius (depth into tissue).
\end{abstract}

Copyright (c) 2007 Quirino Balzano et al. This is an open access article distributed under the Creative Commons Attribution License, which permits unrestricted use, distribution, and reproduction in any medium, provided the original work is properly cited.

\section{INTRODUCTION}

We consider electrically short antennas because, although they are the highest energy density sources, exposure to their fields has not received attention. The absorption of radiofrequency fields in tissue from antennas has been studied with respect to a number of different applications, including communications and telemetry [1-4]. Most of this work has focused on antennas that are designed to radiate energy into tissue, which typically employ resonant half-wavelength designs [5]. We presently consider near-field exposures from electrically short antennas that, without proper matching, are inefficient radiators due to their reactive impedance. However, the reactive fields near the antennas can be strong and possibly cause a significant localized exposure. Exposure to body tissues from electrically short antennas may arise with various communications and telemetry antennas, including antennas used for wireless body area networks. We suggest that some exposure situations involving accidental placement close to the body of circuit elements driven by high-impedance sources can also be approximated by models based on short dipoles. In some important respects, electrically short antennas present different exposure characteristics than resonant dipole antennas. For short antennas, the locations of maximum RF current and maximum charge concentrations are separated by less than one-quarter wavelength along the antenna, in contrast to resonant antennas.
The reactive near fields from such antennas can be higher than for resonant dipoles with the same feedpoint current. This paper addresses the resulting question of how much exposure the near fields from such antennas might produce in tissues.

The specific absorption rate (SAR) produced in tissue from short dipoles has been studied numerically by several authors [6-8]. An even more extreme exposure situation, near-field exposures from Hertzian dipoles (infinitesimal current segments), has recently been examined by Kurniawan et al. [9]. There remains a lack of a general discussion of exposure to tissues from short dipole antennas. We presently consider exposure from electrically short (0.03$0.1 \lambda$ ) insulated dipole antennas that are embedded within tissue. This study has two parts. First, we present an approximate analytical solution of the field equations for an insulated short dipole embedded in tissue and compare its results with those from a finite difference time domain (FDTD) numerical method. Second, we consider the heating that such near-field exposures produce in tissue, using finite element calculations of heat transport.

\section{ANALYTICAL MODEL}

We consider an insulated, electrically short center-fed antenna of length $2 L$ and radius $a$ inside a cylindrical lossless dielectric shell of radius $d$ and infinite length, surrounded by 
a medium whose dielectric are properties characteristics of tissue (Figure 1).

The current $I(y)$ at a distance $y$ from the feedpoint for electrically short antennas is approximately [10]

$$
I(y)=I \sin k_{p}(L-|y|) / \sin k_{p} L, \quad 0 \leq y \leq L,
$$

where $k_{p}=2 \pi / \lambda_{p}$ is the propagation constant of waves in the dielectric shell ( $d \ll L \ll \lambda_{p}, \lambda_{p}=$ wavelength in the dielectric shell). For an electrically short antenna, this can be approximated by a triangular function with height $I$ at the center (the feedpoint of the dipole).

The axial electric and magnetic fields in the outside medium are given by [11]:

$$
\begin{aligned}
H_{\theta}(y, z)=\frac{I}{4 \pi j} \int_{-\infty}^{\infty} J_{0}\left(a \sqrt{k_{p}^{2}-\zeta^{2}}\right) H_{1}^{(2)}\left(z \sqrt{k_{p}^{2}-\zeta^{2}}\right) k_{p} \\
\quad \times\left(\cos \zeta L-\cos k_{p} L\right) / \sqrt{k_{p}^{2}-\zeta^{2}} e^{-j \zeta y} d \zeta, \\
E_{y}(y, z)=\frac{-I \eta_{p}}{(4 \pi)} \int_{-\infty}^{\infty} J_{0}\left(a \sqrt{k_{p}^{2}-\zeta^{2}}\right) H_{0}^{(2)}\left(z \sqrt{k_{p}^{2}-\zeta^{2}}\right) \\
\times\left(\cos \zeta L-\cos k_{p} L\right) e^{-j \zeta y} d \zeta,
\end{aligned}
$$

where $J_{0}$ is a Bessel function of the first kind and order zero, and $H_{0}^{(2)}$ and $H_{1}^{(2)}$ are Hankel functions of order 0 and 1 . In the equation above, $z$ is the radial coordinate (the $\rho$ coordinate in [11]), $\zeta$ is the axial propagation wavenumber, and $\eta_{p}$ is the characteristic impedance of the plastic medium.

With this approximation, the electric fields in the dissipative tissue for $z \geq d$ can be written as follows (see the appendix):

$$
\begin{gathered}
E_{y}(y, z)=\frac{-j \eta_{d} k_{p} \bar{I}}{4 \pi k_{d}}\left[F^{-}(y, z)+F^{+}(y, z)\right. \\
-2 \cos k_{p} L e^{\left.-j k_{d} \sqrt{(z-d)^{2}+y^{2}} / \sqrt{z^{2}+y^{2}}\right],} \\
\begin{aligned}
E_{z}(y, z)=\frac{\eta_{d} k_{p} \bar{I}}{4 \pi k_{d} z}[ & (y-L) F^{-}(y, z) \\
& +(y+L) F^{+}(y, z)-2 y \cos \left(k_{p} L\right) \\
& \left.\times e^{-j k_{d} \sqrt{(z-d)^{2}+y^{2}}} / \sqrt{z^{2}+y^{2}}\right],
\end{aligned}
\end{gathered}
$$

where $F^{-}(y, z)=e^{-j k_{d} \sqrt{(z-d)^{2}+(y-L)^{2}}} / \sqrt{z^{2}+(y-L)^{2}}, F^{+}(y, z)$ $=e^{-j k_{d} \sqrt{(z-d)^{2}+(y+L)^{2}}} / \sqrt{z^{2}+(y+L)^{2}}$, and $\bar{I}$ is related to the impressed current amplitude; $\eta_{d}$ is the characteristic impedance of tissue (see the appendix). For the short dipoles considered here, the maximum SAR is not found near the feedpoint (as it is with resonant dipoles) but is located in two peaks removed from the center, where it is dominated by the radial $(z)$ component of the field. The peaks approach the center of the dipole for increasing values of $L$. This radial field component is a reactive near field, and does not propagate energy away from the antenna in the $z$ direction. At the location of

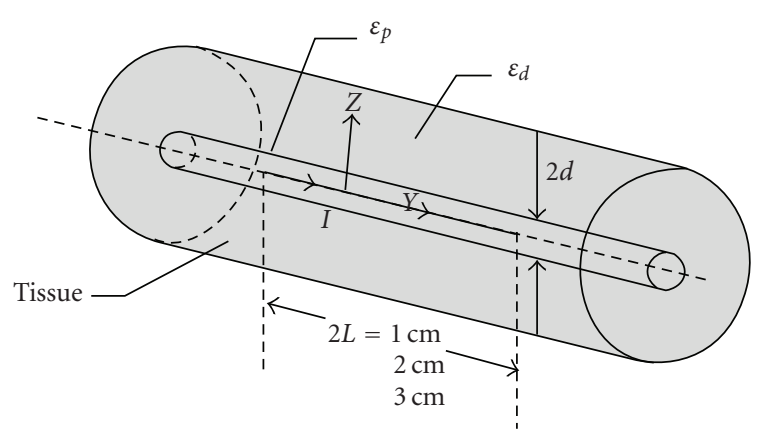

FIGURE 1: Model of antenna, dielectric insulation and dissipative medium.

the maximum SAR, close to the insulating layer $(z \geq d)$, the field magnitude approaches

$$
\left|E_{z}(y, z)\right| \approx 2 y \cos \left(k_{p} L\right) \frac{\eta_{d} k_{p} \bar{I}\left|e^{-j k_{d} \sqrt{y^{2}+(z-d)^{2}}}\right|}{4 \pi k_{d} z^{2} \sqrt{1+(y / z)^{2}}},
$$

with $y<L$ and $z \geq d$. In (5), the radial (and dominant) field component $E_{z}$ falls off with distance $z$ through the combination of two effects. The exponential factor in (5) describes the exponential attenuation of a spherical wave in the tissue, with a skin depth of about $4 \mathrm{~cm}$ at $900 \mathrm{MHz}$. The other space attenuation factors containing $z$ in (5) are a consequence of the near-field exposure. As a consequence, $E_{z}$ falls off rapidly with the radial distance from the antenna (and much more rapidly than for a plane wave at the same frequency). In an exact model, the current $I$ would be modified from the feedpoint current by reflections within the dielectric layer. However, if the dielectric layer is electrically very thin (as it is in the present example), reflections are insignificant and $I$ can be taken as equal to the feedpoint current. If the insulation layer is not electrically very thin, $I$ can be treated as a free input parameter that can be set by limiting the maximum SAR in the tissue or the maximum SAR averaged over a given volume.

The radiation resistance of the antenna is found by dividing the total power radiated by the modulus square of the current [12]. In this case, the total radiated power is given by

$$
\begin{aligned}
\int_{S} \vec{E} \times \vec{H}^{*} \cdot \vec{n} d S & =\int_{0}^{2 \pi} d \int_{-\infty}^{\infty} E_{y}(y, d) H_{\theta}^{*}(y, d) d y d \theta \\
& =d 2 \pi \int_{-\infty}^{\infty} E_{y}(y, d) H_{\theta}^{*}(y, d) d y
\end{aligned}
$$

where $\vec{n}$ is the external normal to the surface of the lossless dielectric cylinder of radius $d$.

From (3) and (A.12), it is possible to evaluate the radiation resistance of the antenna, which admits a functional representation in terms of Bessel functions. The details of the computations are given in the appendix.

From the perspective of hazard assessment, the usual quantity of interest is the SAR, which is the power dissipated per kilogram of tissue. However, for thermal hazards, the biologically significant quantity is the temperature increase, 


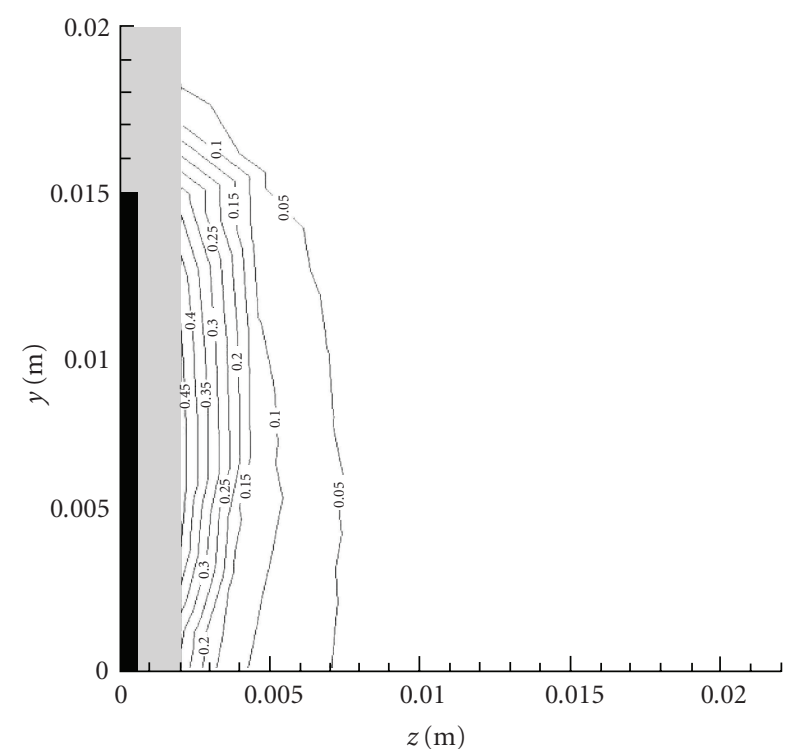

FIGURE 2: SAR pattern of the $3 \mathrm{~cm}$ dipole. Because of symmetry, only half of the antenna is shown; the feedpoint at the center is the origin of the coordinate axes.

not directly the SAR pattern. A detailed thermal model, including effects of blood vessels that might be present near the antennas, is feasible to develop for specific exposure situations.

To provide upper-limit estimates of the temperature increase in a real tissue exposed to short antennas, we presently consider a simplified thermal model, based on the Fourier heat conduction equation that ignores the effects of blood flow;

$$
k \nabla^{2} T+\rho \operatorname{SAR}=\rho C \frac{\partial T}{\partial t},
$$

where the thermal parameters are chosen to be similar to those of soft tissues as follows:

$$
\begin{aligned}
& T=\text { temperature increase above background }(\mathrm{K}), \\
& t=\text { time }(\mathrm{s}), \\
& \rho=\operatorname{density}\left(1000 \mathrm{~kg} \mathrm{~m}^{-3}\right), \\
& C=\text { heat capacity }\left(4000 \mathrm{~m}^{2} \mathrm{sec}^{-2} \mathrm{~K}^{-1}\right), \\
& k=\text { thermal conductivity }\left(0.3 \mathrm{~kg} \mathrm{msec}^{-3} \mathrm{~K}^{-1}\right) .
\end{aligned}
$$

This approximate approach is justified by the very shortrange nature of the SAR, which leads to large thermal gradients near the antenna, and consequently, to dominance of heat conduction in the transport of heat from the region of the antenna.

\section{NUMERICAL MODEL}

Numerical calculations, of both the electromagnetic and heat conduction problems, were performed for dipoles of lengths $2 L$ of 1,2 , and $3 \mathrm{~cm}$, excited at their center at $900 \mathrm{MHz}$. These calculations employed a cylindrical model in which the dipole was surrounded by a lossless cylindrical shell of thickness $2 \mathrm{~mm}$, with dielectric permittivity $\varepsilon_{p}=3$, embedded in an infinite medium whose dielectric properties were assumed

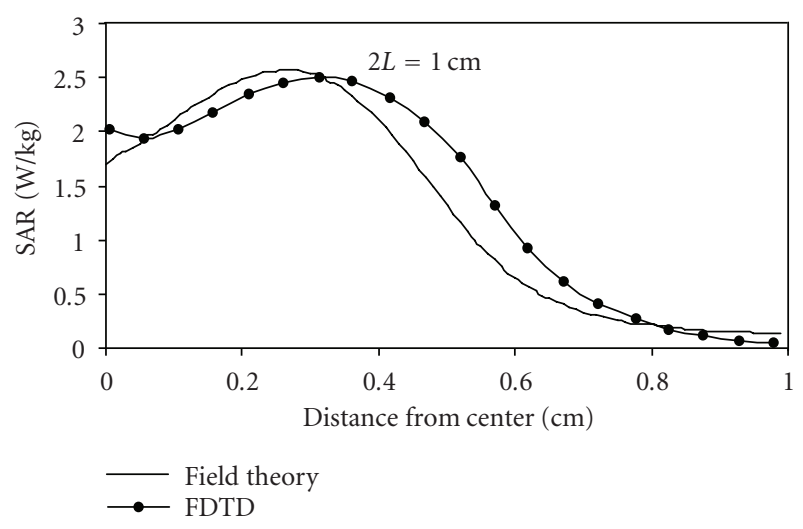

FIGURE 3: SAR at surface of the tissue for the $1 \mathrm{~cm}$ total length antenna.

to be representative of those of soft tissue at $900 \mathrm{MHz}\left(\varepsilon_{d}=\right.$ 54 relative permittivity and $\sigma=1.3 \mathrm{~S} / \mathrm{m}$ conductivity). The $\mathrm{SAR}$ in the tissue around the dipoles was calculated using a finite difference time domain (FDTD) code (FDTD Lab, Motorola Inc. and Tbilisi State University, GA, USA) with a discretization size of $0.5 \mathrm{~mm}$. All results were normalized to a feedpoint current of $10 \mathrm{~mA}$. The calculation used either a fixed number of steps (8000) or terminated when a stability of $0.1 \%$ was reached, In most cases, 8000 steps were used, each step is 1.16 picoseconds, corresponding to a total of about seven cycles at $900 \mathrm{MHz}$.

To provide an upper-limit estimate of the temperature increase, we solved the heat conduction equation using the computed SAR. The heat conduction equation (7) was solved numerically using the computed SAR values using a finite element program (FlexPDE, PDE Solutions, Sunol, CA, USA) to calculate the transient, and steady-state temperature increases in the medium surrounding the antenna. The thermal properties of the insulation layer were chosen to be the same as those of polyethylene.

\section{RESULTS}

\subsection{SAR produced by short dipoles}

Figure 2 shows a contour plot of the SAR pattern of the $3 \mathrm{~cm}$ dipole calculated using FDTD. (Because of symmetry, the pattern of only one half of the antenna is presented.) The dark cylinder represents the antenna, while the shaded box is the insulation. The very short range of the SAR pattern, due to the reactive nature of the fields, is clearly visible from the absence of the peak SAR at the maximum of the $\mathrm{H}$ field and the short depth of penetration in tissue.

The SAR patterns in tissue at the dielectric boundary (along the surface of the antenna insulation) are shown in Figures 3-5 for dipole lengths $2 L$ of 1,2 , and $3 \mathrm{~cm}$, comparing the numerical (FDTD) and analytical results ((3) and (4) for $d \ll L$ ) for a feedpoint current $I=10 \mathrm{~mA}$. Since the FDTD simulations include the effects of reflections within the dielectric layer around the antennas, the agreement between the numerically and analytically obtained SAR values shows that the use of the feedpoint current $I_{\mathrm{fp}}$ for $I$ in (5) is 


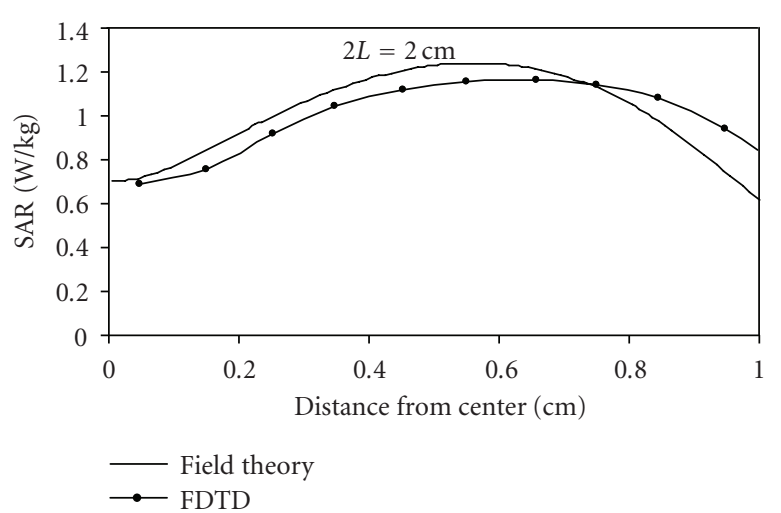

Figure 4: SAR at surface of the tissue for the $2 \mathrm{~cm}$ total length antenna.

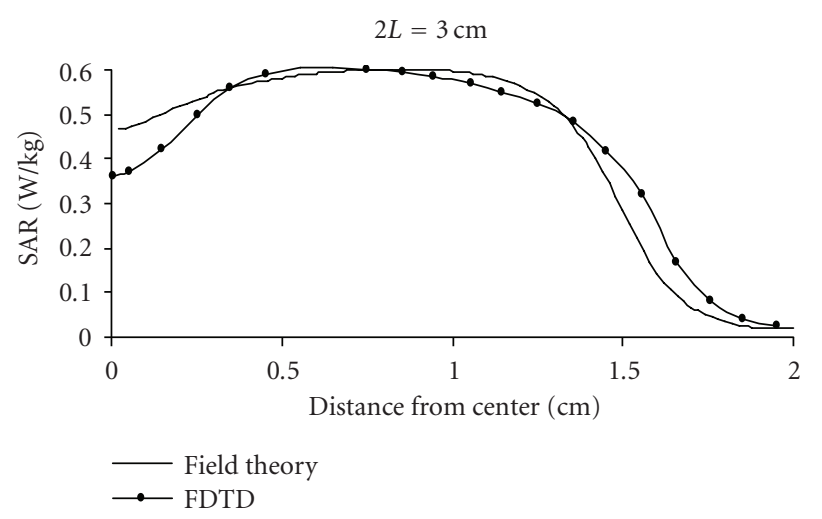

FIgURE 5: SAR at surface of the tissue for the $3 \mathrm{~cm}$ total length antenna.

clearly a good approximation in this case because the simple analytical model predicts correctly the peak SAR values. The differences in the results are due to the simplified analytical equations (3) and (4) of the $E$ fields.

Notice that, in the short dipoles considered here, the peak values of the SAR in the tissue are not at the feedpoint (as they are in resonant dipoles [13]) but are located in pairs symmetrically placed about the feedpoint. As the length of the antenna is increased, these migrate closer to the center of the antenna, the feedpoint.

Figure 6 shows the points of maximum SAR value as a function of radial distance into the tissue making clear the very short range of the SAR for the antennas, as expected from (5).

\subsection{Temperature increase}

Table 1 summarizes the maximum increase in temperature and total deposited power in the tissues from the three antennas (with all results normalized to a feedpoint current of $10 \mathrm{~mA}$ ).

Figure 7 shows the transient temperature increase in the region of maximum SAR in the tissue produced by the three

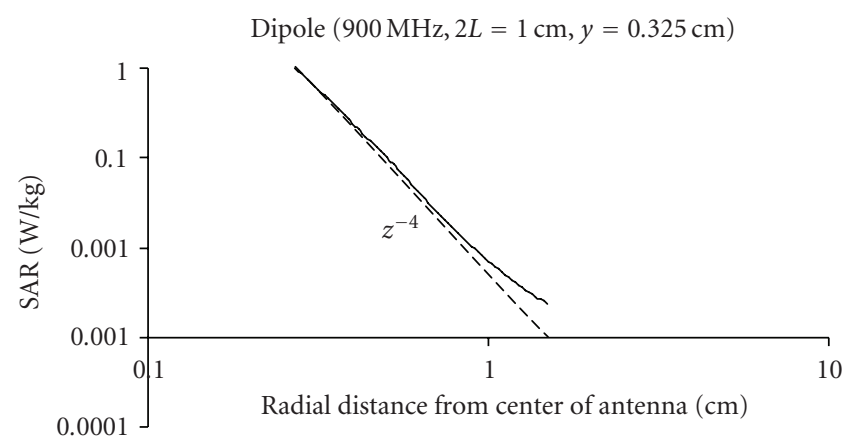

FIGURE 6: Dashed line of slope $z^{-4}$ illustrates approximate slope of the decay in SAR as function of depth into tissue for the $1 \mathrm{~cm}$ total length antenna.

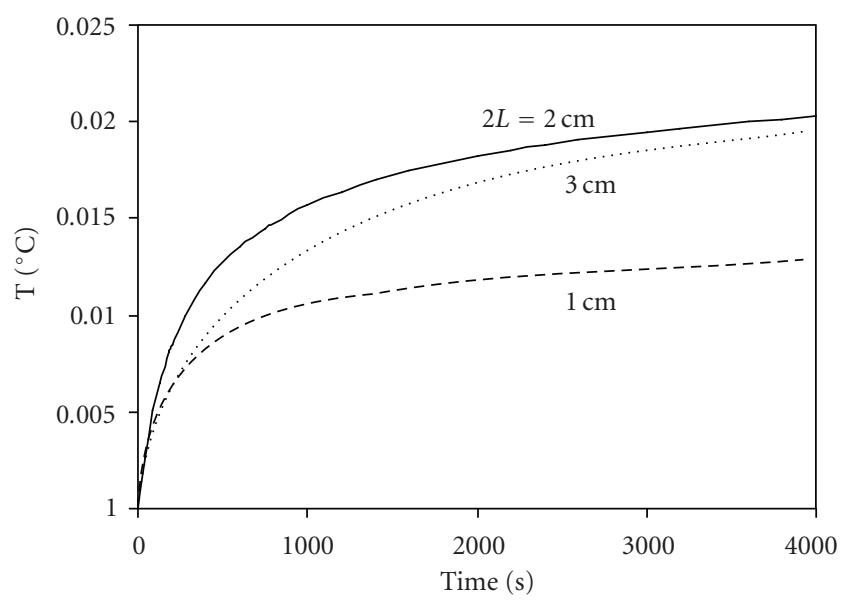

FIGURE 7: Steady-state temperature increases produced at the region of maximum SAR near the three antennas.

antennas. A useful measure of the time needed to approach the steady state is the thermal relaxation time $\tau$, defined as the ratio of the steady-state temperature increase to the initial time rate of increase of temperature:

$$
\tau=\frac{C T_{\mathrm{ss}}}{\mathrm{SAR}_{\max }}
$$

where $T_{\mathrm{ss}}$ is the maximum steady-state temperature increase at the location of the maximum exposure, $\mathrm{SAR}_{\max }$. This relaxation time (Table 1) ranges from 45 to 245 seconds for the three dipoles.

The steady-state pattern of temperature increase for one antenna is shown in Figure 8. Because of the effect of heat conduction, the steady-state temperature increase is spread out over a much larger area than the SAR pattern.

\section{DISCUSSION}

\subsection{Maximum SAR}

Figures 2-5 show that regions of relatively high SAR are present near the surface of the antenna due to reactive fields near the antenna. These fall off very quickly with distance from the antenna. 
TABLE 1: Summary of temperature increases and absorbed power for short dipoles in a tissue-like dielectric medium.

\begin{tabular}{lcccc}
\hline Length, $2 L(\mathrm{~cm})$ & $\begin{array}{c}\text { Maximum SAR in } \\
\text { medium }(\mathrm{W} / \mathrm{kg})^{*}\end{array}$ & $\begin{array}{c}\text { Total power deposited } \\
\text { into medium }(\mathrm{mW})^{*}\end{array}$ & $\begin{array}{c}\text { Maximum temperature } \\
\text { increase in steady state }(\mathrm{K})\end{array}$ & $\begin{array}{c}\text { Thermal relaxation } \\
\text { time } \tau(\mathrm{s})\end{array}$ \\
\hline 1 & 2.5 & 0.5 & 0.028 & 45 \\
2 & 1.2 & 1.4 & 0.043 & 145 \\
3 & 0.5 & 1.2 & 0.028 & 225 \\
\hline
\end{tabular}

${ }^{*}$ Normalized to $I_{\mathrm{fp}}=10 \mathrm{~mA}(900 \mathrm{MHz})$.

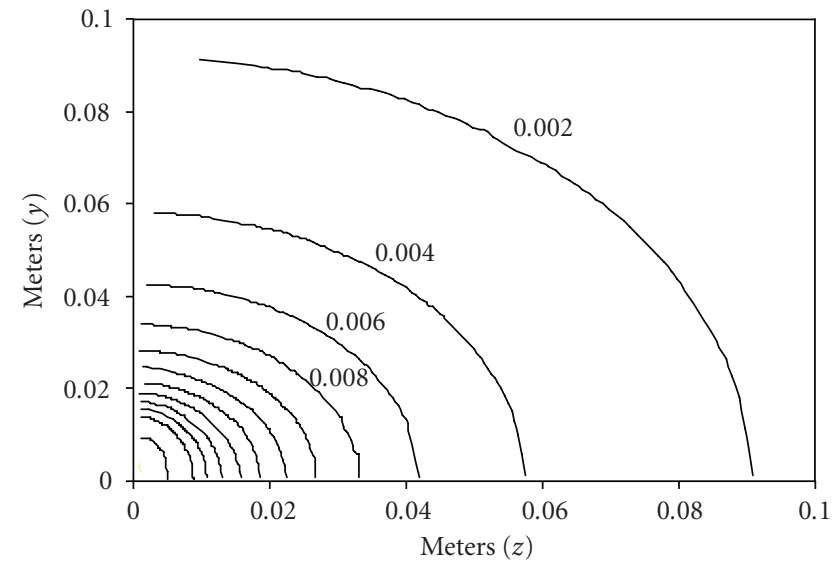

FIGURE 8: Transient temperature increases produced at the region of maximum SAR near the $3 \mathrm{~cm}$ antenna.

It is of interest to compare the maximum SAR produced by these electrically short antennas with resonant dipoles. A useful point of reference is a correlation equation from Kuster and Balzano [13] that provides the maximum SAR beneath a dipole antenna placed a distance $\mathrm{d}$ above a tissue plane:

$$
\begin{aligned}
\operatorname{SARmax} & =\frac{\sigma}{\rho} \frac{\mu \omega}{\sqrt{\sigma^{2}+\varepsilon^{2} \omega^{2}}}\left(1+c_{\mathrm{corr}} \gamma_{\mathrm{pw}}\right) \frac{I_{\mathrm{fp}}}{(2 \pi d)^{2}}, \\
c_{\mathrm{corr}} & =1, \quad d \geq 0.08 \lambda / \gamma_{\mathrm{pw}} ; \\
c_{\mathrm{corr}} & =\sin \left(\frac{\pi d \gamma_{\mathrm{pw}}}{2 \times 0.08 \lambda}\right), \quad d<0.08 \lambda / \gamma_{\mathrm{pw}} .
\end{aligned}
$$

In the above expression, $c_{\text {corr }}$ is a correction factor, $d$ the distance from the dipole to the model, $\gamma_{\mathrm{pw}}$ the plane-wave reflection coefficient for the field, $\lambda$ the free space wavelength, $\rho$ the mass density of the material, $(\varepsilon, \sigma)$ the permittivity and conductivity of the material, and $I_{\mathrm{fp}}$ the antenna current at the feedpoint.

This expression was developed for a different situation (a dipole separated by a distance $d$ above a lossy dielectric plane, with air separating the dipole and the plane), but nevertheless provides a useful point of reference. Using $d=2 \mathrm{~mm}$ (the thickness of the insulation in the antennas presently modeled), (9) yields a maximum SAR of $2.8 \mathrm{~W} / \mathrm{kg}$, which is of the same order of magnitude found in the present study where SAR depends strongly on $L$ in addition to $d$. Using $d=2.8 \mathrm{~mm}$ (an approximation of the electrical thickness of a $2 \mathrm{~mm}$ slab of polyethylene), (9) yields a maximum SAR of
$1.5 \mathrm{~W} / \mathrm{kg}$. We conclude that the maximum SAR produced in the tissue by short dipoles is similar in magnitude to that predicted by the correlation equation (9) developed for resonant dipoles. The total absorbed power in the medium will be far larger with the resonant dipole, however.

\subsection{Temperature increase}

In the steady state (Figure 8), the temperature field extends far more deeply into the medium than the SAR pattern due to effects of heat conduction. This is a consequence of the very short range nature of the SAR produced by these antennas. The heating pattern, after a few seconds, is essentially the same that would be produced by purely surface heating at the insulation-tissue interface, or equivalently by conduction of heat from a heated volume equivalent to that of the antenna and insulation. After an initial thermal transient, the heating pattern hardly depends on the details of the SAR distribution but rather on the total power deposited in the tissue and rate of heat conduction.

This is illustrated by a simple analytical model. Consider an infinite medium whose thermal properties are those given below (7), subject to uniform heating in a spherical region of radius $a$ as follows:

$$
\mathrm{SAR}=S, \quad r \leq a,=0, \quad r>a .
$$

The analytical solution of the heat conduction equation for this problem is elementary. The maximum temperature increase in the center of the region, $T_{\max }$, in the steady state, is

$$
T_{\max }=\frac{S a^{2} \rho}{2 k}=\frac{3 P}{8 \pi a k},
$$

where $P=(4 / 3) \pi a^{3} \rho S$ is the total power deposited in the heated region. For a total power of $1 \mathrm{~mW}$ deposited in a spherical region of $5 \mathrm{~mm}$ radius (comparable to the volume of the cylindrical space heated by the $3 \mathrm{~cm}$ dipole), this corresponds to a maximum temperature increase inside the heated region of about $0.032 \mathrm{~K}$ in the steady state. This is very close to the maximum steady state temperature calculated for the $3 \mathrm{~cm}$ long dipole.

Heat conduction is clearly very efficient in limiting the temperature increase produced by the very short range pattern of RF power deposition produced by these antennas. In a real tissue, the effects of blood perfusion would further reduce the temperature increase.

The threshold for thermal damage to tissue corresponds to local temperature increases of several degrees, which 
would require feedpoint currents in excess of $100 \mathrm{~mA}$ in the present antennas. Currents of this magnitude would be unlikely to be present in practically operating antennas for ordinary applications. Moreover, if such currents were present, the heating effects due to ohmic losses in the conductor [14], and even minimal dielectric losses in the insulation might well exceed those due to near-field exposures in tissue itself.

While these results are particular to the antennas and excitation frequency considered in this study, it would be straightforward to extend these estimates to other antennas and operating frequencies. It would be a useful extension to develop a set of operating parameters, in terms of insulation thickness, antenna length, feedpoint current that would provide practical guidance for safe operation of short dipoles, and other circuit elements in close proximity to tissue, but that is beyond the scope of the present study.

\section{APPENDIX}

\section{A. DERIVATION OF CYLINDRICAL FIELD FUNCTIONS}

From [15], the vector potential from a finite wire in the dielectric $(z>d)$ is given by

$$
A_{y}(y, z)=I /(8 \pi j) \int_{-\infty}^{\infty} F\left(\zeta^{2}, d\right) H_{0}^{(2)}\left(z \sqrt{k_{d}^{2}-\zeta^{2}}\right) e^{-j \zeta y} d \zeta
$$

where $F\left(\zeta^{2}, d\right)$ is a function to be determined by enforcing the continuity of the tangential fields at $z=d$.

The three EM field components for $z \geq d$ are as follows:

$$
\begin{aligned}
& E_{y}(y, z)=\eta_{d} /\left(j k_{d}\right)\left(k_{d}^{2}-\partial^{2} / \partial y^{2}\right) A_{y}(z, y) ; \\
& E_{z}(y, z)=\eta_{d} /\left(j k_{d}\right) \partial^{2} / \partial y \partial z A_{y}(y, z) ; \\
& H_{\theta}(y, z)=-\partial / \partial z A_{z}(y, z) .
\end{aligned}
$$

From (A.1)-(A.2) of the main text, the stationary fields in the plastic sheath for $a \leq z \leq d$ are expressed by

$$
\begin{aligned}
E_{y}(y, z)= & -I \eta_{p} /(4 \pi) \int_{-\infty}^{\infty} J_{0}\left(a \sqrt{k_{p}^{2}-\zeta^{2}}\right) \\
& \times\left[H_{0}^{(2)}\left(z \sqrt{k_{p}^{2}-\zeta^{2}}\right)+\Gamma\left(\zeta^{2}, d\right) H_{0}^{(1)}\left(z \sqrt{k_{p}^{2}-\zeta^{2}}\right)\right] \\
& \times\left(\cos \zeta L-\cos k_{p} L\right) e^{-j \zeta y} d \zeta, \\
H_{\theta}(y, z)= & I /(4 \pi j) \int_{-\infty}^{\infty} J_{0}\left(a \sqrt{k_{p}^{2}-\zeta^{2}}\right) \\
& \times\left[H_{1}^{(2)}\left(z \sqrt{k_{p}^{2}-\zeta^{2}}\right)-\Gamma\left(\zeta^{2}, d\right) H_{1}^{(1)}\left(z \sqrt{k_{p}^{2}-\zeta^{2}}\right)\right] \\
& \times k_{p}\left(\cos \zeta L-\cos k_{p} L\right) / \sqrt{k_{p}^{2}-\zeta^{2}} e^{-j \zeta y} d \zeta,
\end{aligned}
$$

where $H_{0}^{(2)}, H_{1}^{(2)}, H_{0}^{(1)}$, and $H_{1}^{(1)}$ are Hankel functions, $I$ the amplitude of the current on the wire, and $\Gamma\left(\zeta^{2}, d\right)$ the reflection coefficient at the dielectric boundary.
Forcing the continuity of the fields for $z=d$, one finds

$$
\begin{aligned}
F\left(\zeta^{2}, d\right)= & 2 k_{d} \eta_{p} /\left(\eta_{d} k_{p}\right) J_{0}\left(a \sqrt{k_{p}^{2}-\zeta^{2}}\right) \\
& \times\left[H_{0}^{(2)}\left(d \sqrt{k_{p}^{2}-\zeta^{2}}\right)+\Gamma\left(\zeta^{2}, d\right) H_{0}^{(1)}\left(d \sqrt{k_{p}^{2}-\zeta^{2}}\right)\right] \\
& \times k_{p} / H_{0}^{(2)}\left(d \sqrt{k_{d}^{2}-\zeta^{2}}\right)\left(\cos \zeta L-\cos k_{p} L\right) \\
/ & \left(k_{d}^{2}-\zeta^{2}\right), \\
\Gamma\left(\zeta^{2}, d\right)= & {\left[k_{p} / \sqrt{k_{p}^{2}-\zeta^{2}} H_{1}^{(2)}\left(d \sqrt{k_{p}^{2}-\zeta^{2}}\right)\right.} \\
& / H_{1}^{(2)}\left(d \sqrt{k_{d}^{2}-\zeta^{2}}\right)-k_{d} \eta_{p} / \eta_{d} \sqrt{k_{p}^{2}-\zeta^{2}} \\
& \left.\times H_{0}^{(2)}\left(d \sqrt{k_{p}^{2}-\zeta^{2}}\right) / H_{0}^{(2)}\left(d \sqrt{k_{d}^{2}-\zeta^{2}}\right)\right] \\
/ & H_{0}^{(1)}\left(d \sqrt{k_{p}^{2}-\zeta^{2}}\right) / H_{0}^{(2)}\left(d \sqrt{k_{d}^{2}-\zeta^{2}}\right) \\
& \left.+H_{1}^{(1)}\left(d \sqrt{k_{p}^{2}-\zeta^{2}}\right) / H_{1}^{(2)}\left(d \sqrt{k_{d}^{2}-\zeta^{2}}\right)\right] .
\end{aligned}
$$

Note that if $k_{d}=k_{p}, \Gamma\left(\zeta^{2}, d\right)=0$, giving the function $F\left(\zeta^{2}, d\right)$ the form

$$
\begin{aligned}
F\left(\zeta^{2}, d\right)= & 2 k_{d, p} J_{0}\left(a \sqrt{k_{p, d}^{2}-\zeta^{2}}\right) \\
& \times\left(\cos \zeta L-\cos k_{p, d} L\right) /\left(k_{p, d}^{2}-\zeta^{2}\right),
\end{aligned}
$$

which inserted in (A.1) gives the equation of the vector potential of a dipole radiating in the space with dielectric permeability $\varepsilon_{p, d}$.

It is worth mentioning that the term $J_{0}\left(a \sqrt{k_{p, d}^{2}-\zeta^{2}}\right)$ is a differential operator [10] and its contributions become relevant only for $y, z<a$, so we set $J_{0}\left(a \sqrt{k_{p, d}^{2}-\zeta^{2}}\right)=1$ in the following considerations.

The function

$$
C\left(\zeta^{2}, d\right)=\left[H_{0}^{(2)}\left(d \sqrt{k_{p}^{2}-\zeta^{2}}\right)+\Gamma\left(\zeta^{2}, d\right) H_{0}^{(1)}\left(d \sqrt{k_{p}^{2}-\zeta^{2}}\right)\right]
$$

part of $F\left(\zeta^{2}, d\right)$, is well behaved (no poles or singularities) along the real values of $\zeta$.

If we consider the case $d \ll \lambda_{p}$ (wavelength in the dielectric insulation) and evaluate the limit $d \rightarrow 0$ we have

$$
\lim _{d \rightarrow 0} C\left(\zeta^{2}, d\right) \approx H_{0}^{(2)}\left(d \sqrt{k_{p}^{2}-\zeta^{2}}\right)
$$

If we call $\bar{I}=I\left(\eta_{p} / \eta_{d} k_{p} \sin k_{p} L\right)$, then the electric field components (A.2) and (A.3) are expressed by

$$
\begin{aligned}
E_{y}(y, z)= & -\eta_{d} \bar{I} /(8 \pi) \int_{-\infty}^{\infty} H_{0}^{(2)}\left(z \sqrt{k_{d}^{2}-\zeta^{2}}\right) \\
& \times F(d, \zeta) 2 k_{p}\left(\cos \zeta L-\cos k_{p} L\right) e^{-j \zeta y} d \zeta,
\end{aligned}
$$




$$
\begin{aligned}
E_{z}(y, z)= & j \eta_{d} \bar{I} /(8 \pi) \int_{-\infty}^{\infty} H_{1}^{(2)}\left(z \sqrt{k_{d}^{2}-\zeta^{2}}\right) F(d, \zeta) \\
& \times 2 k_{p}\left(\cos \zeta L-\cos k_{p} L\right) / \sqrt{k_{d}^{2}-\zeta^{2}} \zeta e^{-j \zeta y} d \zeta
\end{aligned}
$$

with $F(d, \zeta)=H_{0}^{(2)}\left(d \sqrt{k_{p}^{2}-\zeta^{2}}\right) / H_{0}^{(2)}\left(d \sqrt{k_{d}^{2}-\zeta^{2}}\right)$.

From (A.10), the formulas for the $E$ fields in the dissipative medium are found by direct integration [16] and relation [17];

$$
\begin{aligned}
& 1 /(2 j) \int_{-\infty}^{+\infty} H_{1}^{(2)}\left(\rho \sqrt{k^{2}-\zeta^{2}}\right) / \sqrt{k^{2}-\zeta^{2}} e^{-j \zeta z} d \zeta \\
& \quad=j /(k \rho) e^{-j k \sqrt{\rho^{2}+z^{2}}} .
\end{aligned}
$$

The magnetic field is

$$
\begin{aligned}
H_{\theta}(y, z)= & 2 k_{p} \bar{I} /(8 \pi j) \int_{-\infty}^{\infty} H_{1}^{(2)}\left(z \sqrt{k_{d}^{2}-\zeta^{2}}\right) \\
& \times F(d, \zeta)\left(\cos \zeta L-\cos k_{p} L\right) / \sqrt{k_{d}^{2}-\zeta^{2}} \zeta e^{-j \zeta y} d \zeta \\
= & -j \bar{I} /(4 \pi z) \\
& \times\left[e^{-j k_{d} \sqrt{(z-d)^{2}+(y-L)^{2}}}+e^{-j k_{d} \sqrt{(z-d)^{2}+(y+L)^{2}}}\right. \\
& \left.\quad 2 \cos k_{p} L e^{-j k_{d} \sqrt{(z-d)^{2}+y^{2}}}\right] .
\end{aligned}
$$

The radiation resistance of the short antennas is given by

$$
R_{r}=d 2 \pi /|\bar{I}|^{2} \operatorname{Re}\left(\int_{-\infty}^{\infty} E_{y}(y, d) H_{\theta}^{*}(y, d) d y\right) .
$$

The integrals in (A.13) can be computed using the relation [18]

$$
\int_{0}^{\infty} e^{-a t} / \sqrt{1+t^{2}} d t=\pi / 2\left[\mathbf{H}_{0}(a)-Y_{0}(a)\right],
$$

where $\mathbf{H}_{0}(a)$ is a function of Struve, and $Y_{0}(a)$ is a Bessel function of the second kind, both of order 0 . The final result is

$$
\begin{aligned}
R_{r}= & \operatorname{Re}\left(1 / \sqrt{\varepsilon_{d}}\right) 377 \sqrt{\varepsilon_{p}} / 16 \\
\times & {\left[\left(1+2 \cos ^{2} k_{p} L\right)+\cos \beta L e^{-2 \alpha L}-4 \cos \beta L \cos k_{p} L\right] } \\
\times & {\left[\mathbf{H}_{0}(2 \alpha L)-Y_{0}(2 \alpha L)\right]+\operatorname{Re}\left(1 / \sqrt{\varepsilon_{d}}\right) 377 \sqrt{\varepsilon_{p}} / 16 } \\
\times & {\left[e^{-2(\alpha-j \beta) L} \int_{0}^{2 L / d} e^{-j 2 \tau \beta d} / \sqrt{1+\tau^{2}} d \tau\right.} \\
& \left.+4 \cos k_{p} L e^{-j \beta L-\alpha L} \int_{0}^{L / d}\left(e^{-2 \tau \alpha d}-e^{-j 2 \tau \beta d}\right) / \sqrt{1+\tau^{2}} d \tau\right],
\end{aligned}
$$

where $\alpha=\operatorname{Re}\left(-j k_{d}\right)$ and $\beta=\operatorname{Im}\left(-j k_{d}\right)$.

For $2 L=2 \mathrm{~cm}, R_{r} \approx 13 \Omega$, and for $2 L=3 \mathrm{~cm}, R_{r} \approx 12 \Omega$. As expected, $\lim _{L \rightarrow 0} R_{r} \rightarrow 0$.

\section{ACKNOWLEDGMENTS}

This research is supported in part by the Mobile Manufacturers Forum and Motorola Inc. The authors are grateful to Dr. Giorgi Bit-Babik of Motorola Corporate Electromagnetic Research Laboratory, Plantation, FL, USA for guidance on FDTD methods and to Ms. Bo Yang of the University of Maryland Electrical and Computer Engineering Department, College Park, MD, USA for her skillful preparation of the figures.

\section{REFERENCES}

[1] J. R. Wait, Electromagnetic Radiation from Cylindrical Structures, Pergamon Press, New York, NY, USA, 1959.

[2] R. W. P. King, "Electromagnetic field generated in model of human head by simplified telephone transceiver," Radio Science, vol. 30, no. 1, pp. 267-281, 1995.

[3] J. Nadobny, R. Pontalti, D. Sullivan, et al., "A thin-rod approximation for the improved modeling of bare and insulated cylindrical antennas using the FDTD method," IEEE Transactions on Antennas and Propagation, vol. 51, no. 8, pp. 1780-1796, 2003.

[4] A. Hirata, M. Fujimoto, T. Asano, J. Wang, O. Fujiwara, and T. Shiozawa, "Correlation between maximum temperature increase and peak SAR with different average schemes and masses," IEEE Transactions on Electromagnetic Compatibility, vol. 48 , no. 3 , pp. 569-578, 2006.

[5] K. M. Jones, J. A. Mechling, J. W. Strohbehn, and B. S. Trembly, "Theoretical and experimental SAR distributions for interstitial dipole antenna arrays used in hyperthermia," IEEE Transactions on Microwave Theory and Techniques, vol. 37, no. 8, pp. 1200-1209, 1989.

[6] O. Kivekäs, T. Lehtiniemi, and P. Vainikainen, "On the general energy-absorption mechanism in the human tissue," $\mathrm{Mi}$ crowave and Optical Technology Letters, vol. 43, no. 3, pp. 195201, 2004.

[7] A. Christ, T. Samaras, A. Klingenböck, and N. Kuster, "Characterization of the electromagnetic near-field absorption in layered biological tissue in the frequency range from $30 \mathrm{MHz}$ to $6000 \mathrm{MHz}$," Physics in Medicine and Biology, vol. 51, no. 19, pp. 4951-4965, 2006.

[8] M. Klemm and G. Troester, "EM energy absorption in the human body tissues due to UWB antennas," Progress in Electromagnetics Research, vol. 62, pp. 261-280, 2006.

[9] T. Kurniawan, A. W. Wood, and R. L. McIntosh, "Near field and dosimetry of RF bioeffects research," in Proceedings of the Workshop on Applications of Radio Science, Leura, New South Wales, February 2006.

[10] E. C. Jordan and K. G. Balmain, Electromagnetic Waves and Radiating Systems, Prentice-Hall, Englewood Cliffs, NJ, USA, 2nd edition, 1968.

[11] Q. Balzano, O. Garay, and K. Siwiak, "The near field of dipole antennas, part 1: theory," IEEE Transactions Vehicular Technology, vol. 30, no. 4, pp. 161-174, 1981.

[12] R. F. Harrington, Time-Harmonic Electromagnetic Fields, McGraw-Hill, New York, NY, USA, 1961.

[13] N. Kuster and Q. Balzano, "Energy absorption mechanism by biological bodies in the near field of dipole antennas above 300 MHz," IEEE Transactions on Vehicular Technology, vol. 41, no. 1, pp. 17-23, 1992.

[14] K. Gosalia, J. Weiland, M. Humayun, and G. Lazzi, “Thermal elevation in the human eye and head due to the operation of a retinal prosthesis," IEEE Transactions on Biomedical Engineering, vol. 51, no. 8, pp. 1469-1477, 2004.

[15] R. F. Harrington, Time-Harmonic Electromagnetic Fields, McGraw-Hill, New York, NY, USA, 1961. 
[16] L. Felsen and N. Marcuvitz, Radiation and Scattering of Waves, Prentice-Hall, Englewood Cliffs, NJ, USA, 1973.

[17] V. A. Ditkin and A. P. Prudnikov, Integral Transforms and Operational Calculus, Pergamon Press, London, UK, 1965.

[18] N W McLachlan, Bessel Functions for Engineers, Oxford University, London, UK, 2nd edition, 1961.

\section{AUTHOR CONTACT INFORMATION}

Quirino Balzano: Department of Electrical and Computer

Engineering, University of Maryland, College Park, MD 20742, USA; qbalzano@eng.umd.edu

Kenneth R. Foster: Department of Bioengineering, School of Engineering and Applied Science, University of Pennsylvania, 210 S. 33rd Street, Philadelphia, PA 19104-6392, USA;

kfoster@seas.upenn.edu

Asher R. Sheppard: Asher Sheppard Consulting, 108 Orange Street, Suite 8, Redlands, CA 92373, USA; Department of Physiology and Pharmacology, School of Medicine, Loma Linda University, 11021 Campus Street, Loma Linda, CA 92350, USA; ashersheppardconsulting@verizon.net 

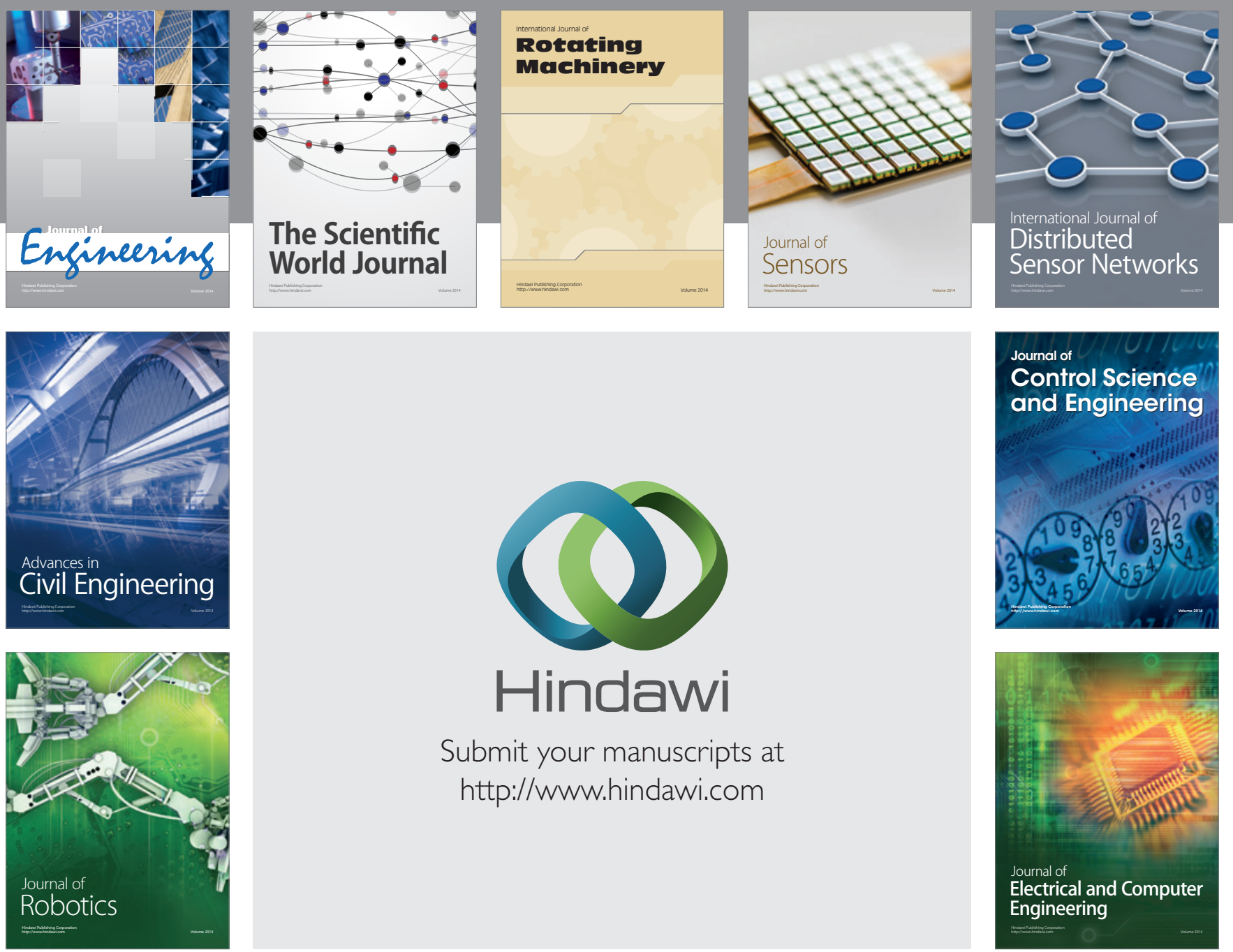

Submit your manuscripts at

http://www.hindawi.com
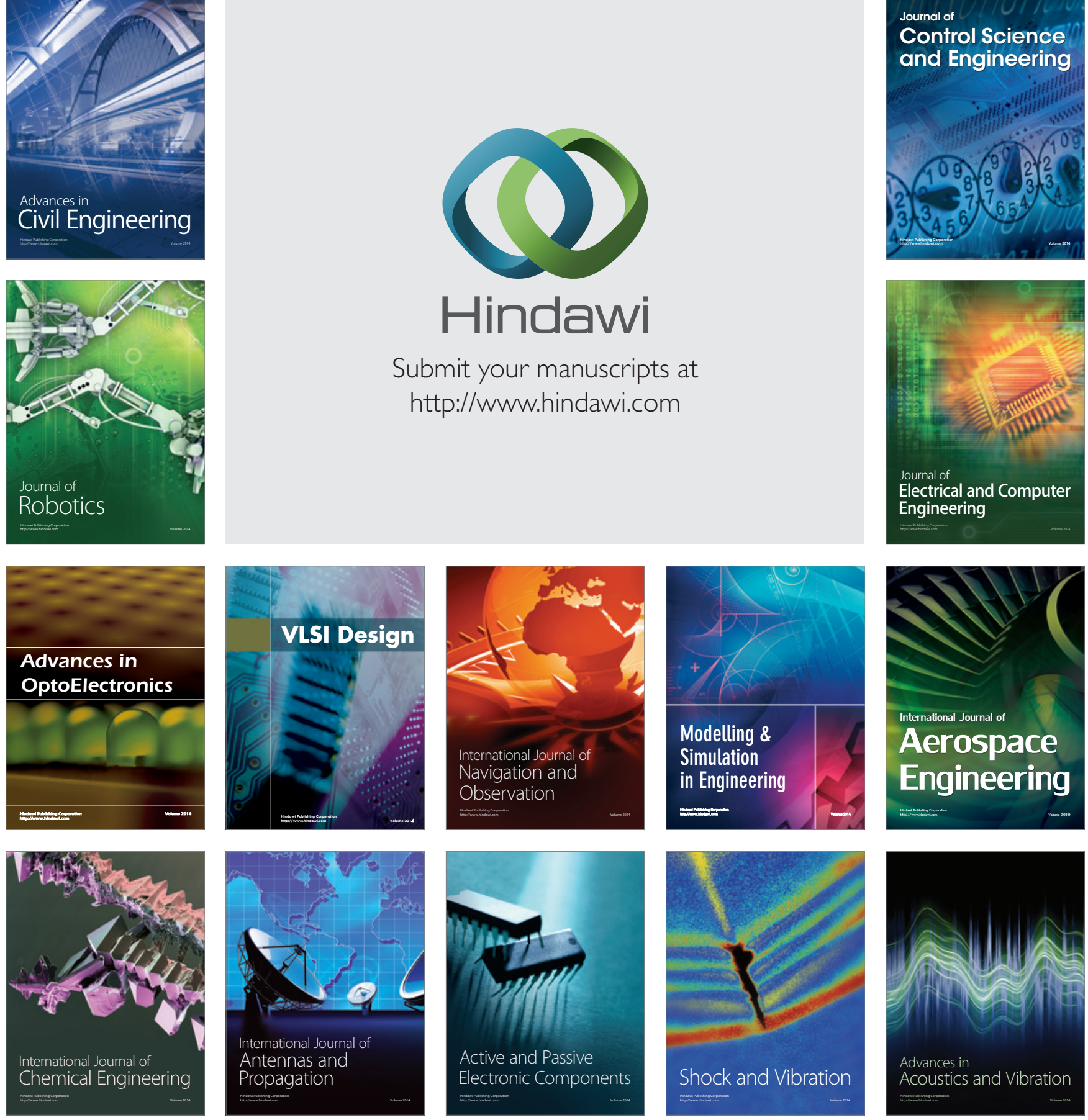